\title{
Analysis of integrated position navigation of intelligent scraper based on Calman filtering technology
}

\author{
Yong Jiang ${ }^{1, a}$, Hao Zhang ${ }^{2, b}$ and Hengtong $\mathrm{Li}^{1, \mathrm{c}}$ \\ ${ }^{1}$ Beijing General Rerearch Institute of Mining \& Metallurgy, Beijing,100160, China \\ ${ }^{2}$ University of Science and Technology Beijing, Beijing,100083,China \\ ajiangyong23@163.com, 'bhanghaojy138@163.com, ${ }^{\circ}$ lihengtong1994@163.com
}

Keywords: Intelligent scraper, Calman filtering technology, Positioning and navigation.

Abstract: In this paper, with the intelligent scraper as the research object, studies the application of integrated position navigation algorithm of intelligent scraper by Calman filtering technology, and to achieve the optimal combination of positioning and navigation information fusion, pressing the data of dead reckoning solution process system and laser ranging system to the process of positioning solution at the same time. The simulation results show that the positioning error federated Calman filtering algorithm can effectively reduce positioning error of the integrated system, improve the positioning accuracy of the system.

\section{Introduction}

Calman filtering technology is one of the estimation algorithms for random signal. It introduced the concept of the state space of modern control theory in the filtering technology, and system is described by state equation. With the recurrence calculation, it do not need to know the value of measurement of the past, and only according to the measure of a moment ago value and the current value, then it can calculate the recursive estimation of the current value[1,2,3].

\section{CombinedCalman filtering algorithm}

Combined Calman filtering algorithm of estimated the unrelated conditions by each filter. If the sub filter estimate is independent. For the two local filter $(\mathrm{N}=2)$, a local state estimation for $\hat{X}_{1}$ and $\hat{X}_{2}$, and corresponding error of the estimation is $P_{11}$ and $P_{22}$. Considering the $X_{k}$ global state estimation mixed is a linear combination of the local state estimation, i.e.

$$
\hat{X}_{g}=W_{1} \hat{X}_{1}+W_{2} \hat{X}_{2}
$$

$W_{1} 、 W_{2}$ - The weighted matrixto be determined.

The global state estimation $\hat{X}_{g}$ should meet the following two conditions:

if the local state estimation which is $\hat{X}_{1}$ and $\hat{X}_{2}$ is unbiased estimation, then the global state estimation $\hat{X}_{g}$ is unbiased estimation, i.e.

$$
E\left[X-\hat{X}_{g}\right]=0
$$


the variance matrix of estimation error of $\hat{X}_{g}$ is minimum, i.e.

$$
P_{g}=E\left[\left[X-\hat{X}_{g}\right]\left[X-\hat{X}_{g}\right]^{T}\right.
$$

From the above assumptions can be introduced that

$$
\begin{aligned}
& P_{g}=\left[P_{11}^{-1}+P_{22}^{-1}\right]^{-1} \\
& \hat{X}_{g}=\left[P_{11}^{-1}+P_{22}^{-1}\right]^{-1}\left(\left[P_{11}^{-1} \hat{X}_{1}+P_{22}^{-1} \hat{X}_{2}\right)\right.
\end{aligned}
$$

Using mathematical induction, the above results can be extended to the $\mathrm{N}$ cases of a local state estimation.

$$
\begin{aligned}
& P_{g}=\left(\sum_{i=1}^{N} P_{i i}^{-1}\right)^{-1} \quad\left(P_{i j}=0, i \neq j\right) \\
& X_{g}=P_{g} \sum_{i=1}^{N} P_{i i}^{-1} \hat{X}_{i}
\end{aligned}
$$

Combined Calman filtering algorithm of estimated the related conditions by each filter.In general, all the filters are related, therefore, can make the appropriate transformation for the filtering process using variance upper bound, making the local filtering estimation in fact not related. Setting the state estimation formula of each filter is expressed as follows:

$$
\hat{X}_{i}=\left[\begin{array}{c}
\hat{X}_{a i} \\
\hat{X}_{b i}
\end{array}\right]
$$

$\hat{X}_{a i}$ - Estimation of each sub filter public state $\hat{X}_{a}$;

$\hat{X}_{b i}$ - The $i$ filter proprietary state estimation.

Assuming that distributed the information of noise amount system process into each local filter and the main filter, i.e.

$$
Q^{-1}=\sum_{i=1}^{N} \lambda_{i} Q_{i}^{-1}+\lambda_{m} Q_{m}^{-1}
$$

According to the principle of conservation of information, we can know:

$$
\sum_{i=1}^{N} \lambda_{i}+\lambda_{m}=1
$$

The initial information of state estimation $P_{0}^{-1}$ can also be assigned according to the method. If the information can be the same distribution state estimation, i.e. 


$$
P^{-1}=P_{1}^{-1}+P_{2}^{-1}+L P_{N}^{-1}+P_{m}^{-1}=\sum_{i=1}^{N} \lambda_{i} P^{-1}+\lambda_{m} P^{-1}
$$

\section{Integrated position navigationbased on Calman filtering technology}

Intelligent scraper complete autonomous navigating walking mainly rely on the detection of environmental information of roadway tunnel wall combined with electronic map matching in the underground tunnel. Through the fusion of multi sensors information and filtering algorithm with Calman, system estimated the state and direct the scraper travelling. As shown in Figure 1 for the graph of information fusion algorithm of intelligent algorithm scraper.

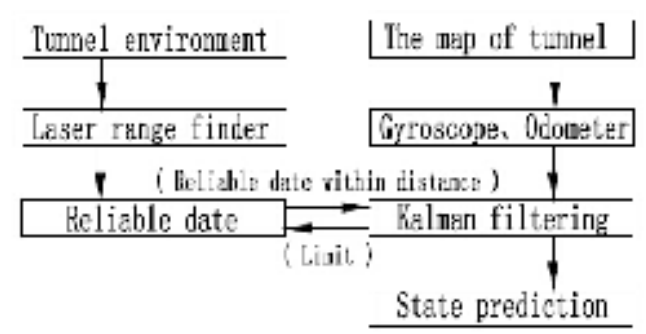

Fig. 1 The graph of information fusion algorithm

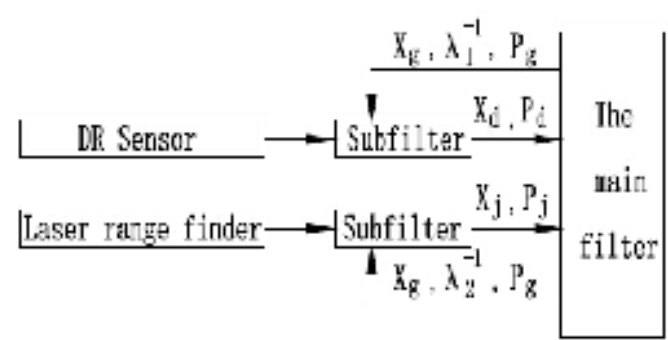

Fig. 2 Combined with Calman filter structure of integrated positioning

Design of combined with Calman filter structure of Dead reckoning / Laser ranging recognition integrated navigation as shown in figure 2. Combined system consists of dead reckoning system, laser ranging recognition subsystem and main filter based on three components. Two subsystems each has a local Calman filter, used to process detecting dataof dead reckoning system and laser rangefinder / recognition system.Inputting the local state estimation in $X_{d}, X_{j}$ of subsystem, and the matrix $P_{d}, \quad P_{j}$ of estimation error covarianceto the main filter and the information of local filter was analyzed and mixed by main filter, and the optimal estimated $X_{g}, P_{g}$ of the whole system are output. And at the same time according to the information distribution coefficient $\lambda_{1}, \lambda_{2}$ of a local filter is reset and feedback, as the initial value of the next time iteration, thus the local filter state estimation precision is also improved.

\section{Simulation of integrated navigation algorithm}

In order to verify the reckoning and laser rangefinder / landmark recognition integrated navigation algorithm, we made the computer simulation for the real route data of the vehicle, dead reckoning system location data and laser rangefinder / recognition system of location data, and then analyzed the performance of integrated navigation algorithm[4,5]. As shown in Figure 3 and Figure 4 are respectively given the curve in lateral displacement error of positioning recognition alone for scraper of dead reckoning positioning system and laser ranging positioning system, and Figure 5 is the curve in lateral displacement error of integrated positioning system of scraper.

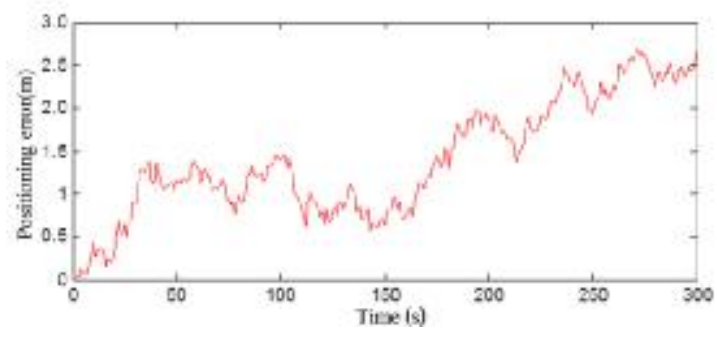

Fig. 3 The curve in lateral displacement error of dead reckoning positioning system

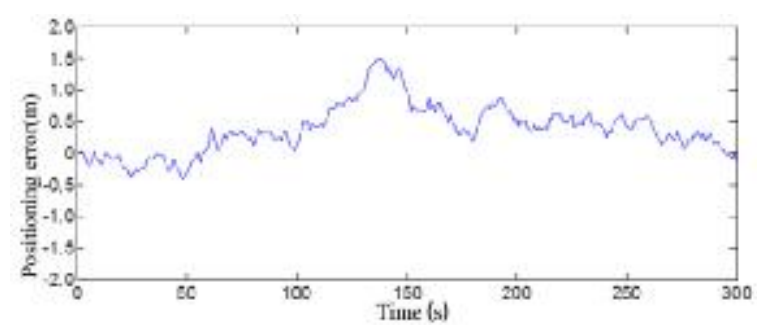

Fig. 4 The curve in lateral displacement error of laser ranging positioning system 


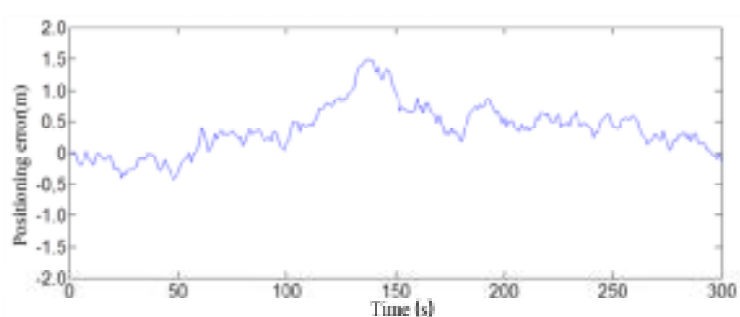

Fig. 5 The curve in lateral displacement error of integrated positioning system

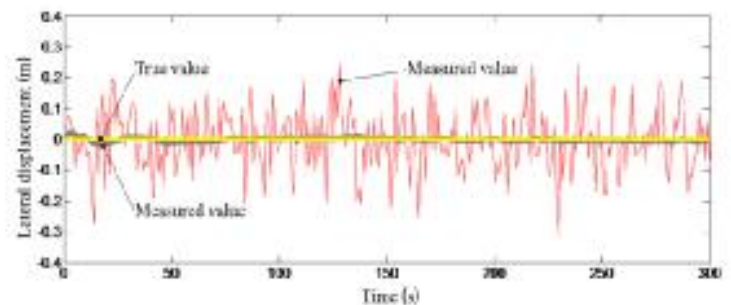

Fig. 6 Estimated position of system after Calman filtering processing

In order to further narrow the positioning error of the integrated navigation system, so that the positioning accuracy need to further improve. The control of the scraper should be more accurate, and the travel route is more reasonable, and does not appear the abnormal vibration of steering appeared in front of the experiment. System simulation is made in this paper for the integrated navigation system using federated Calman filtering algorithm. Figure 6 is the map of estimated positionof system afterCalman filtering processing.

\section{Conclusion}

(1)positioning error of alone dead reckoning or Laser ranging location is relatively large.After the combined positioning of dead reckoning and laser ranging identification, the positioning error is significantly reduced, and the error fluctuations is more smooth, so the positioning accuracy is improved significantly.

(2)Calman filter can effectively make good estimates of the true value in the relatively large disturbances. Even if the observation is added noise (such as when the tunnel wall is very irregular), Calman filter can filter effectively, and estimate the state of scraper accurately. The positioning error can be effectively reduced for the combined system using federated Calman filtering algorithm, and it can improve the positioning accuracy of the system.

\section{Acknowledgements}

This work was financially supported by National high technology research and development plan of "863" project (2011AA060403).

\section{References}

[1] Chen Yao,Li Yinong,Han Jiawei.GPS/INS integrated navigation particle filter based on structural decomposition,J.Chinese Journal of Automotive Engineering.2015,5(01):16-22.

[2] Xiong Jian,LIU Jian-ye,Lai Ji-zhou,Zhou Zhai-he.State Estimation of In-Wheel Motor Electric Vehicle Based on Extended Kalman Filter,J. Control and Decision.2012,27(06):957-960.

[3] Wu Jinyuan,Fang Jiancheng, Yang Zhaohua.ASUKF based relative navigation method for mars probe using pulsar,J.Chinese Journal of Scientific Instrument.2013,34(08):1711-1716.

[4] Joseph Nsasi Bakambu, Vladimir Polotski. Autonomous System forNavigation and Surveying innderground Mines. Journal of Field Robotics, 2007, 24(10), 829-847.

[5]H. Martinez-Barbera, D.Herrero-Perez. Autonomous navigation of anautomated guided vehicle in industrial environments. Robotics and Computer-Integrated Manufacturing. 2010, 26: 296-311. 